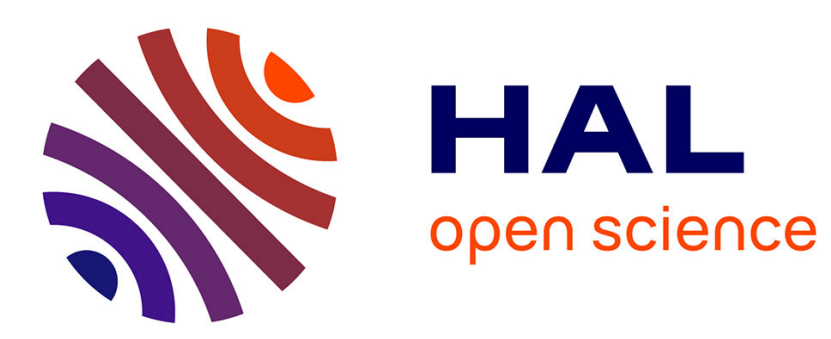

\title{
A Redundancy based Protocol for Safety Message Dissemination in Vehicular Ad Hoc Networks
} Imen Achour, Tarek Bejaoui, Anthony Busson, Sami Tabbane

\section{To cite this version:}

Imen Achour, Tarek Bejaoui, Anthony Busson, Sami Tabbane. A Redundancy based Protocol for Safety Message Dissemination in Vehicular Ad Hoc Networks. The 82 nd IEEE Vehicular Technology Conference, IEEE, Sep 2015, Boston, United States. hal-01242275

\section{HAL Id: hal-01242275 \\ https://inria.hal.science/hal-01242275}

Submitted on 11 Jan 2016

HAL is a multi-disciplinary open access archive for the deposit and dissemination of scientific research documents, whether they are published or not. The documents may come from teaching and research institutions in France or abroad, or from public or private research centers.
L'archive ouverte pluridisciplinaire HAL, est destinée au dépôt et à la diffusion de documents scientifiques de niveau recherche, publiés ou non, émanant des établissements d'enseignement et de recherche français ou étrangers, des laboratoires publics ou privés. 


\title{
A Redundancy-based Protocol for Safety Message Dissemination in Vehicular Ad Hoc Networks
}

\author{
Imen Achour ${ }^{* \#}$, Tarek Bejaoui ${ }^{*}$, Anthony Busson ${ }^{\S}$,Sami Tabbane ${ }^{*}$ \\ *Mediatron Lab. SupCom, University of Carthage, Tunisia \\ ${ }^{\#}$ Telnet Innovation Labs. Telnet Holding, Tunisia \\ ${ }^{\S}$ Laboratory of Parallel Computing, University of Lyon 1 - ENS - Inria, France
}

\begin{abstract}
The diversity of applications' types in vehicular ad hoc networks (VANETs) has spawned a large variety of messages that need to be disseminated in vehicle to vehicle (V2V) communication mode. The most critical messages are those dedicated for safety applications such as accident warning, road hazardous warning, signal violation warning, etc. The dissemination of this sort of messages is a challenging task in VANETs since they should be efficiently transmitted i.e. by achieving high packet delivery within a limited transmission delay and an acceptable overhead. In this work, we propose a robust and an original data dissemination protocol called "Redundancy-based Protocol (RBP)". Unlike most of densitybased protocols, RBP is beaconless. It takes into account the surrounding vehicle density during the broadcasting process through a specific metric, named "Packet Redundancy Ratio", calculated locally at each vehicular node. On the basis of this metric, each vehicle is able to dynamically define the probability of rebroadcast in order to mitigate the broadcast storm problem. The simulation results show that the proposed protocol outperforms the slotted 1-persistence scheme in terms of packet drop ratio, and network load while still maintaining a low Endto-End delay and high packet reachability. This scheme is suitable either for safety applications or for further kinds of applications by saving the network capacity consumption.
\end{abstract}

Keywords-Vehicular Ad hoc Networks; Broadcast storm; Slotted 1-persistence; Vehicle Density; Data Dissemination; Vehicle to Vehicle Communication

\section{INTRODUCTION}

Vehicular Ad-hoc Networks (VANETs) are emerging as new prominent technologies for improving the efficiency and the safety of intelligent transportation systems (ITSs). Composed of mobile vehicles connected via wireless links, VANETs leads to a wide variety of applications ranging from safety and traffic management to generalized infotainment and entertainment applications.

Safety applications are considered as the most critical and valuable applications category. They are meant to address passenger safety, which is considered the highest priority for ITS. Yet, in order to ensure road safety, different safety alerting messages[1] [2] (e.g., Collision Risk Warning, Traffic hazard warnings, Vehicle type warning, etc.) need to be efficiently disseminated between mobile nodes in a vehicle-to-vehicle (V2V) communication mode. Nevertheless, safety data dissemination is remaining a challenging issue in VANETs. Considering the wireless environment of VANET, these messages are typically disseminated through a broadcast technique. As an intuitive solution, messages may be broadcasted in a blind flooding scheme. Thereby, upon receiving a message, each vehicle must rebroadcast immediately the same message in order to ensure the data reachability for distant vehicles situated in the area of interest. However, this technique is not scaled with the network density. Indeed, in a dense networks flooding becomes very costly in terms of medium occupancy due to an excessive redundant broadcasts which results in serious contention and collision [3]. This is typically referred to as a broadcast storm problem [4].

To cope with this problem, several "broadcasting suppression techniques" have been proposed in the literature . The common solution employed by most techniques is to select a set of vehicles as relay nodes with the aim of reducing the excessive number of redundant messages.These solutions are essentially classified into delay-based, probability-based and hybrid protocols. The set of criteria considered for the relaying node selection are mainly the distance between the sender and the receiver, the vehicle direction with relation to the message direction and the surrounding vehicles' density.

In this context, we propose a novel hybrid (delay and probability) dissemination protocol named "Redundancy Based Protocol (RBP)". .RBP is a robust protocol dealing with two criteria which are the distance and the density of surrounding vehicles. The aim of this protocol is to achieve a high packet reachability within an acceptable transmission delay while reducing the network resource consumption. On one hand, the delay based technique in RBP is inspired from the philosophy of "Slotted 1-Persistence" disseminating protocol, as denoted in [4]. On the other hand, RBP proposes a new design for the probability scheme that takes into account the vehicle density. This is considered as the main contribution of this work. In fact, contrary to most of the density based protocols, RBP is beaconless. Based on its "Redundancy Ratio" metric, each vehicle is aware of the surrounding vehicles' density and able to dynamically tune the probability of rebroadcast. Indeed, this metric has shown its direct relation with the vehicles' density. More the vehicles' density increases, more the broadcasting storm problem is severe and subsequently the redundancy ratio.

The reminder of this paper is organized as follows. In Section II, we report on previous works. Section III describes our proposed Redundancy Based Protocol in detail. Section IV, is dedicated for presenting the simulation environment and 
discuss the performance evaluation. Finally, concluding remarks and future works are presented in Section V.

\section{RELATED WORKS}

Various techniques dealing with data dissemination issue, in particular data broadcast, in vehicular environment have been proposed in literature. These techniques were reviewed in different works [6][7][8]. Some of them are defined for vehicle to infrastructure communication (V2I), whereas, some others are designed for a $\mathrm{V} 2 \mathrm{~V}$ communication, without considering the road infrastructure. In dense network, $\mathrm{V} 2 \mathrm{~V}$ data dissemination is facing the so-called "Broadcast storm Problem".

In order to mitigate this problem, researchers have proposed some suppression techniques. In [5], three basics suppression strategies are presented to be run in the Network layer: a delay based technique denoted "slotted 1-persistence", a probabilistic based technique called "weighted p-persistence, and a hybrid scheme "slotted p-persistence". Based on the simulation results presented in [5], "slotted 1-persistence" disseminating protocol (S1PD) outperforms the two others protocols by reducing the number of excessive redundant rebroadcast while achieving a low end-to-end delay and a high packet reachability. The idea behind S1PD is, given a fixed number of time slots, the farthest vehicles from the transmitter vehicle (from where the message has been originated), will be assigned to the earliest time slot, i.e. will be given the shortest waiting time, to rebroadcast. Thereby, vehicles within other time slots would have time to cancel their transmissions upon the receipt of the same message during their waiting time. As a result, redundant broadcasts may be suppressed.

However, "slotted1-persistence" technique may suffer from synchronization problem, which has been identified and demonstrated in [9]. This problem can occur when numerous vehicles assigned to a single time slot start their transmissions simultaneously, which may results in a high number of collisions. To this end, authors in [10] propose an optimization of S1PD called "Optimal 1-Persistence Dissemination" protocol (O1PD). O1PD copes with the synchronization problem by adding a small delay to the time slot in the network layer. The time slot in O1PD is assigned to each vehicle according to its distance from the source vehicle and to its moving direction toward the broadcast message direction.

In order to efficiently overcome these problems, some recent works [11-15] have considered the surrounding vehicles' density as a major criteria that should be taken into account during the broadcast process. Indeed, vehicles' density has a significant impact on the protocol performance.

In [11] [12], authors propose further enhancement of O1PD called "Distributed Optimized Time (DOT)". Based on the vehicles' density knowledge within the transmission range, DOT provides the possibility of controlling the number of vehicles belonging to the same time slot. As a result, the irregular distribution of vehicles among time slots is prevented, which leads to control the transmission redundancy. Yet, the number of vehicles that should be assigned to a single time slot is not dynamically defined. Authors in [13], have proposed a broadcast technique by which the rebroadcast probability $\mathrm{P}$ is dynamically controlled according to the vehicle density. Meanwhile, in [14] a dynamic scheme for broadcast is presented to adjust the number of slots according to the vehicles' density.

Unlike our scheme, all the aforementioned density based protocols relay on a periodic exchange of beacon messages for density estimation. Gathering the information contained in beacons enables each vehicle to maintain the table of neighbors' position information. However, in dense and highly dynamic networks, the beacon messages may contribute in increasing collisions and contention, especially if they are not properly coordinated.These messages will lead then to waste bandwidth.

Our work further differs from these protocols, in a focus on increasing the dissemination reliability while using efficiently the limited bandwidth. In particular, thanks to the defined redundancy ratio metric, vehicles transmitters are able to dynamically adjust the probability of rebroadcast according to the surrounding vehicles' density without the need of beacon exchange.

\section{REDUNDANCY BASED PROTOCOL}

In this paper we propose a novel density based dissemination protocol called "Redundancy Based Protocol -. RBP". RBP is a hybrid protocol which combines delay and probability-based dissemination schemes. Basically, the aim of this protocol is to deal with the broadcast storm problem by reducing excessive broadcasts while offering high packet reachability and low end-to-end delay in highway environment. Thus, in addition to the distance parameter used for computing the waiting time, RBP takes into account the vehicles' density to determine its re-broadcast probability. The key feature of our algorithm is that no beacon exchange is required even though the vehicles' density is considered.

To further understand the protocol details, we first propose to describe general assumptions and requirements for a proper operation. Next, we define the "redundancy ratio" and its correlation with the vehicles' density. A,d then, a detailed description of the fundamental steps of RBP will be presented.

\section{A. Assumptions and requirements}

In this work, we assume that no road infrastructure is deployed. Only V2V communications are available to generate and disseminate data. In this context, we assume that each vehicle is equipped with on-board wireless devices in conformity with the available IEEE $802.11 \mathrm{p}$ standard [16]. Moreover, the proposed scheme assumes that every vehicle is able to determine permanently its current geographical position using Global Positioning System (GPS). Furthermore, we require the presence of local application, running on the source vehicle. This application,is responsible of generating and sending a special type of messages named "Decentralized Environmental Notification Message (DENM)" [1]. According to [2], these messages are generated upon the occurrence of an event related to a road hazard (accident) or an abnormal traffic conditions (traffic jam). Such message should be periodically retransmitted at a certain frequency and within a defined period. Throughout the text, we will often simply refer to them as messages or data packets. 


\section{B. Redundancy Metric}

The Redundancy Ratio " $r$ " measures the proportion of the total received messages (original messages + redundant messages) to the number of original received messages (new messages). It is continuously updated by each vehicle during the transmission process. The reset of this metric is triggered when no packet is received during a predefined period of time $\Delta \mathrm{t}$.

Given a fixed number of source vehicles, simulation results have shown in Fig. 1 that the redundancy ratio increases logarithmically by increasing the vehicles' density. This means that the number of received packets by a source packet becomes higher. So, we can conclude that the number of redundant packet increases in accordance with the increase of the vehicles' density. Simulation parameters are not presented here, as we just like to demonstrate the variation on the packet redundancy with relation to the vehicles' density. Simulation parameters and results will be presented in Section IV.

Hence, from this observation we conclude that the probability of rebroadcast in RBP should be inversely proportional to this metric. Then, higher the redundancy ratio, smaller the broadcasting probability is. This leads to reduce the probability of broadcast when the vehicles' density increases.

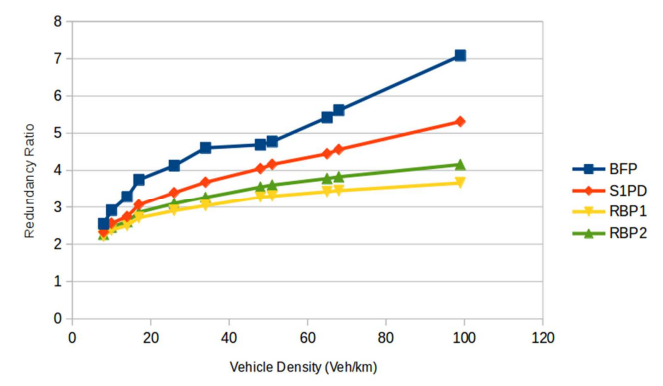

Fig. 1. Redundancy Ratio variation with different vehicles" density

\section{Protocol description}

The main concept of RBP is presented by a flow-diagram in Fig. 2 which can be described as follow:

According to RBP, each message is characterized by a unique ID which consists of the source vehicle's ID and the local packet ID. As well, each vehicle has a data buffer that stores the original data packets (not duplicated ones), either received or generated by the local application running on the transmitter vehicle. Thereby, upon receiving a packet, the vehicle checks first whether the message is known or not, i.e., message's ID is already stored in the data buffer or not. If this is the case, this means that the received message is redundant and should be discarded after updating the redundancy ratio " $r$ " parameter. On the other hand, if the message is original, then it is sent to be stored in the buffer with the label "scheduled for rebroadcast $=$ true". In this way, the message is considered as a potential rebroadcast, to which a waiting time " $\mathrm{W}_{\mathrm{t}}$ " is assigned and a timer is immediately triggered. When the message's timer expires and no redundant message is received from other forwarders during the waiting process, the message is broadcasted with a Broadcast Probability "P". Otherwise, the rebroadcast decision is cancelled.

The suppression techniques adopted in RBP, is inspired from S1PD scheme since it achieves the best performance among the other schemes proposed in [5]. Given a fixed number of time slots " $\mathrm{N}_{\mathrm{t}}$ ", the waiting time is calculated as presented in (1):

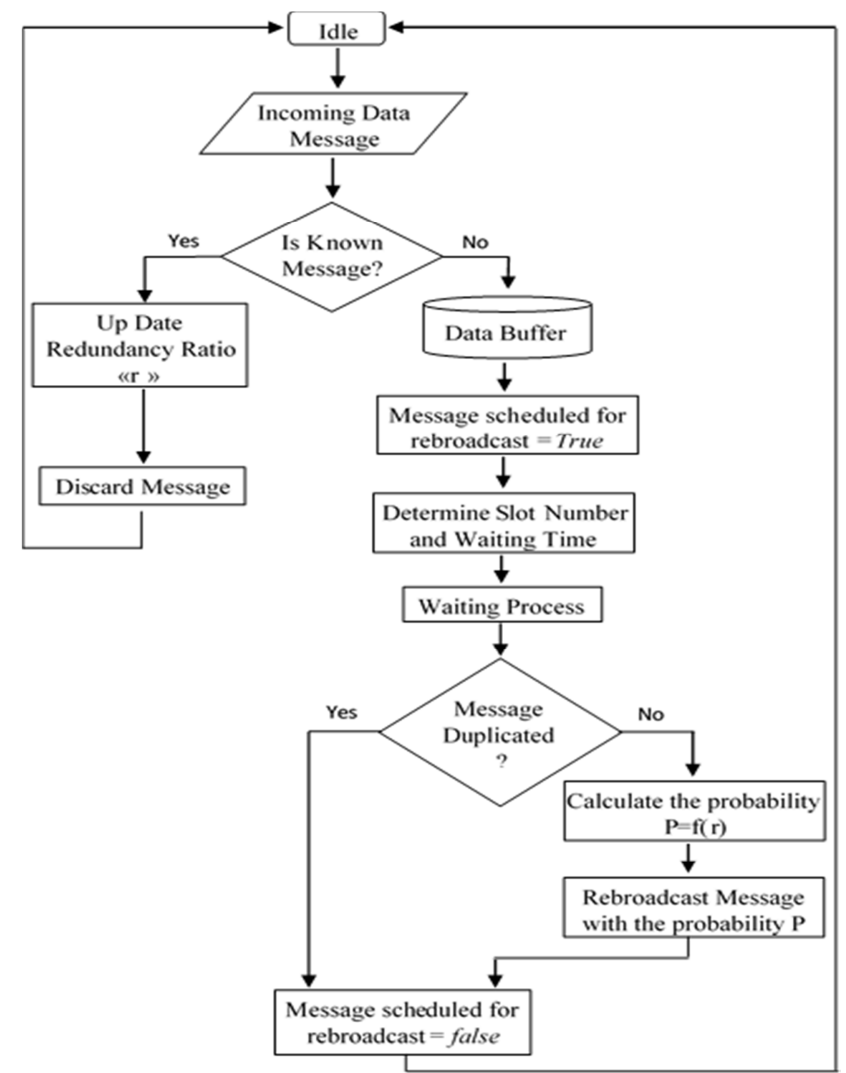

Fig. 2. Flow chart of rebroadcasting procedure for Redundancy Based Protocol

$$
\mathrm{W}_{\mathrm{t}}=\left\lfloor\mathrm{N}_{\mathrm{t}} *\left(1-\frac{\min \left(\mathrm{D}_{\mathrm{ij}}, \mathrm{R}\right)}{\mathrm{R}}\right)\right\rfloor * \delta
$$

Where $D_{i j}$ is the relative distance between the transmitter " $i$ " and the receiver " $\mathrm{j}$ ", $\mathrm{R}$ is the average transmission range and $\delta$ is larger than one hop delay including medium access delay and propagation delay.

Hence, assigning vehicles to different time slots will clearly alleviate the broadcast storm by reducing redundant and simultaneous broadcasts. Though, a similar problem in a small scale can still occur when numerous vehicles are assigned to the same time slot. To cope with this problem we have proposed two ways of calculating the re-broadcast probability "P", based on the surrounding vehicles' density. The first probability given by (2), is a combination between the current redundancy ratio value " $r_{\text {current }}$ ", calculated at the sending time, and the previous one " $r_{\text {prev }}$ " incorporated in the previous calculated probability " $\mathrm{P}_{\text {prev }}$ " for the last sent packet. The main purpose of this correlation, is to continuously regulate the 
redundancy ratio in order to maintain a normalized amount of redundancy.

$$
\mathrm{P}_{1}=\frac{2}{\mathrm{r}} * \mathrm{P}_{\text {prev }}=\frac{2}{\mathrm{r}_{\text {current }}} * \frac{2}{\mathrm{r}_{\text {prev }}}
$$

The second probability is in a direct relation with the current redundancy ratio " $r$ ", calculated just when the message timer expires. This probability follows equation 3 .

$$
\mathrm{P}_{2}=\frac{2}{\mathrm{r}}
$$

In this way, and in both equations, a node forwards a message with a probability, inversely proportional to the redundancy ratio, and thus inversely proportional to the vehicle density. Hence, regions with high density of vehicles will decrease the nodes' suitability to be a forwarding node. Yet, in low dense regions more candidates will be suitable for relaying received message.

\section{PERFORMANCE EVALUATION}

In this section we present the performance evaluation of RBP, carried out by means of extensive simulations, using NS3 [17] simulator. These simulations aim to evaluate the efficiency and the effectiveness of our protocol in a vehicular environment. To this purpose we have used a micro-traffic simulator called "SUMO - Simulation of Urban Mobility" [18] for a realistic mobility trace in a highway environment. We consider a straight three-lanes highway of $6 \mathrm{~km}$ length. We set the bit rate to $6 \mathrm{Mbit} / \mathrm{s}$ in the MAC layer. We adjust the transmission power to achieve roughly 700 meters of transmission range, assuming a Nakagami propagation model. For the suppression technique mechanism, we set $\delta$ to $4 \mathrm{~ms}$ and we fix the total number of time slots $\mathrm{N}_{\mathrm{t}}$ to 7 (time slots per 100 $\mathrm{m})$. For the application scenario, we configure the 5 first vehicles to generate at every second a new message of 500 bytes length. In order to evaluate the RBP scalability, we vary the vehicles' density from 8 vehicles $/ \mathrm{km}$ to 99 vehicles $/ \mathrm{km}$ at the maximum speed of $20 \mathrm{~m} / \mathrm{s}$. Each plotted result is an average of 20 runs of 100 s.

Two disseminating protocols are selected for the performance comparison, namely:

- mBFP: ( modified Blind Flooding Protocol). Upon receiving a packet, the vehicle checks the packet novelty then rebroadcasts it immediately

- S1PD: Upon receiving a packet, the vehicle checks the packet novelty, then rebroadcasts it after the expiration of the assigned waiting time $\mathrm{W}_{\mathrm{t}}$, calculated according to [5].

For the sake of simplicity and til the end of this paper, we mean by RBP1, the proposed protocol using P1, and RBP2, the protocol using $\mathrm{P} 2$.

Our evaluation considers the following metrics:

- $\quad$ Packet Delivery Ratio (PDR): the average number of original packets successfully received by a vehicle, compared to the total number of generated messages.
- Forwarding Ratio (FR): the proportion of vehicles in the network that are involved in the rebroadcast of a source packet.

- End-to-End Delay (E2EDelay): the average difference between the data packet generation time by the source vehicle and the received time of this packet by the last reached vehicle.

- Link Load (bit/s): the average of broadcast traffic (in terms of bits) received by each vehicle over a unit of time.

- $\quad$ Packet Drop Ratio (DROP): the average amount of erroneous received packet of a vehicle compared to the total received packets.

Based on the simulation results shown in Fig. 3, we can notice that forwarding ratio drastically degrades with S1PD and $\mathrm{RBP}$, compared to mBFP. In addition we can observe that the forwarding ratio degrades in S1PD and RBP when the vehicle density increases, while it rises in mBFP. This shows that more the selection of the forwarding vehicle is smarter, more the dissemination performance is better. However, RBP1 presents the best performance since it is able to reduce by $50 \%$ the number of forwarders as compared to S1PD. So, we can say that RBP1 is efficient in terms of reducing the number of packets hop, since lesser vehicles are involved in the reforwarding process to reach the maximum number of vehicles in the network. Also, reducing the number of re-forwarders may have an important impact on the dissemination latency.

Besides, Fig.4 illustrates the fact that RBP1 and RBP2 are able to roughly achieve the same PDR (as it is achieved by S1PD protocol) while reducing the number of forwarding nodes. Thereby, the network resources' consumption will significantly decreases. This observation is further proved through Fig. 5 that compares the link load for RBP1 and RBP2 schemes with that obtained while using S1PD scheme. Here, RBP scheme outperforms S1PD in terms of "link load" for all the vehicles' densities. In particular, RBP1 has reduced the link load about $40 \%$ compared to that of S1PD in high dense network, which illustrates the protocol scalability. This amounts to a substantial decline in the received messages curve (these curves are not shown in this paper due to lack of space). This improvement, leads to save the limited bandwidth and increase the network capacity to allow other applications to be run simultaneously.

Moreover, RBP1 and RBP2 presents in Fig. 6, lesser drop ratio compared to that for S1PD. This may highlight how efficient RBP protocol to mitigate the broadcast storm by more reducing the network contention and collision compared to S1PD, while still achieving a high PDR and a low end to end delay, as show in Fig.7.

Thanks to RBP, each node will be able to adapt its suitability to be a re-forwarding node according to the current state of the network density (through the redundancy ratio). Hence, according to RBP, each node will be able to get most likely the best decision without the need of information and feedbacks from neighboring nodes. As a result, the efficiency of RBP emerges from the adaptive local behavior of each node, since each node is acting on its own. 
On the other hand, the focus on RBP on its own leads to deduce the following statement. As reported, RBP1 outperforms RBP2 in terms of reducing the forwarding ratio and link load, but this is achieved at the cost of a slight loss in terms of PDR, as expected. In reality, P1 attempts to further maintain lesser amount of redundancy compared to P2. Although excessive redundant packets may result in severe contention and collision problems, a certain amount of redundancy should be preserved to guarantee a high packet delivery in a lossy network. Thus, a tradeoff between the cost of transmissions and the need of high reachability should be done according to the applications' requirements.

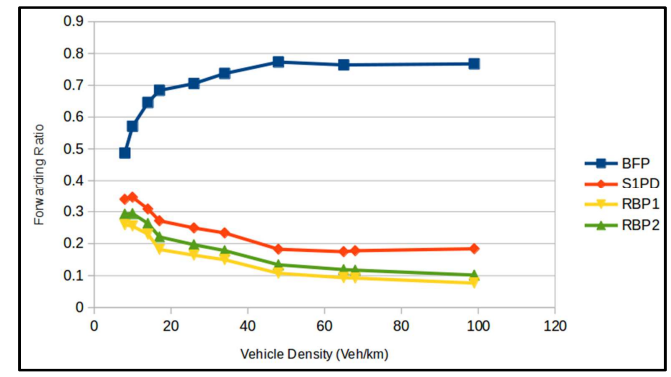

Fig. 3. Forwarding Ratio vs Various Vehicle Density

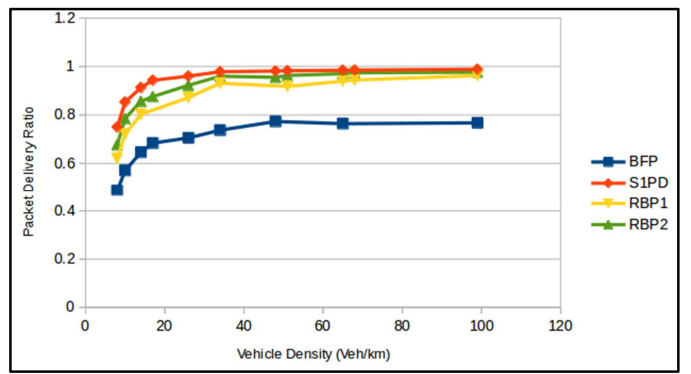

Fig. 4. Packet Delivery Ratio vs Various Vehicle Density

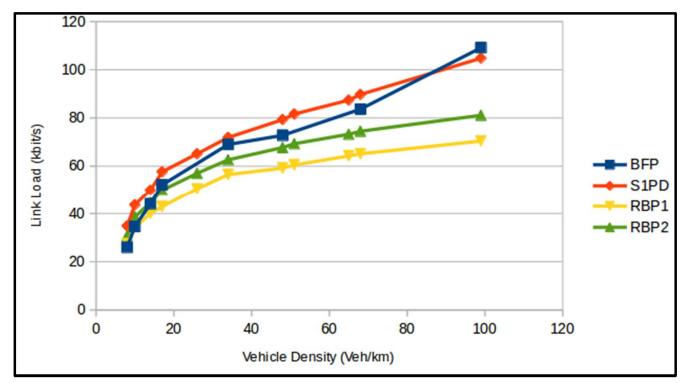

Fig. 5. Link Load vs Various Vehicle Density

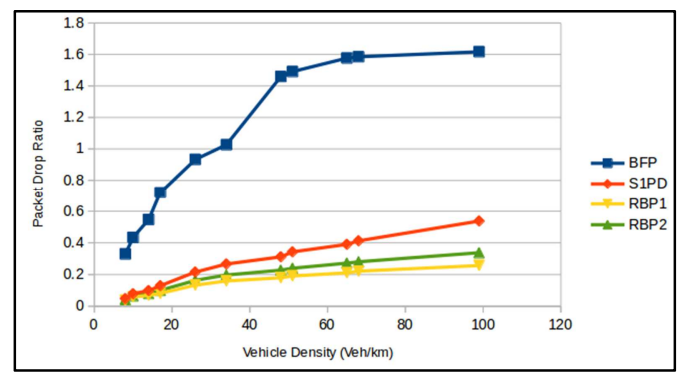

Fig. 6. Packet Drop Ratio vs Various Vehicle Density

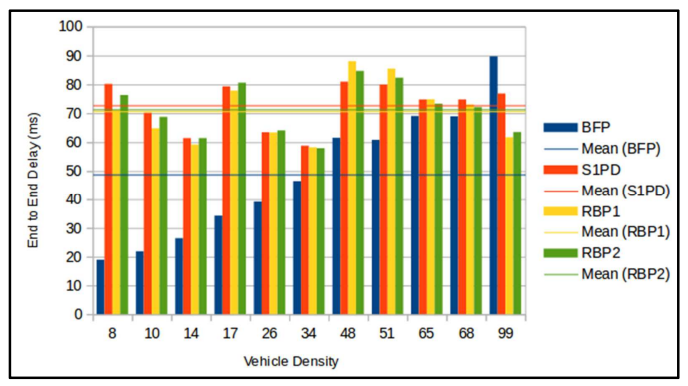

Fig. 7. End to End Delay vs Various Vehicle Density

\section{CONCLUDING REMARKS}

In this work, we propose a density-based protocol for safety message dissemination, called "Redundancy Based Protocol (RBP)". This protocol aims to meet the challenging problems of broadcast storm in scalable network. For this purpose, we designed a beaconless mechanism for density-awareness. Thanks to a particular developed metric, named "Redundancy Ratio", each vehicle is implicitly aware of the surrounding vehicles' density. It will be able then to define its forwarding facility when the waiting time, calculated according to S1PD, expires. This is done in a distributed manner with no need to a beaconing process with neighboring nodes.

Simulation results showed that our scheme outperforms S1PD protocol and achieves high level performance for safety message dissemination, since it provides a high PDR within a low End-to-End delay. RBP is moreover scalable, since the number of broadcast messages (link load) is significantly reduced, and then allows the efficient use of the limited bandwidth.

Future work includes the accommodation of RBP to sparse networks and the investigation of the connectivity problem between communicating vehicles. Furthermore, the redundancy ratio may be further handled in order to adaptively adjust the amount of redundancy in accordance with the requirements of each VANET application.

\section{ACKNOWLEDGMENT}

This work is a part of the MOBIDOC project achieved under the PASRI program, funded by the European Union and administered by the ANPR.

\section{REFERENCES}

[1] ETSI, T. Intelligent transport systems (ITS); vehicular communications; basic set of applications; definitions (Vol. 1). Tech. Rep. ETSI TR $102638,2009$.

[2] ETSI, T.Intelligent Transport Systems (ITS); Vehicular Communications; Basic Set of Applications; Part 3: Specifications of Decentralized Environmental Notification Basic Service. ETSI EN 302 637-3, 2014.

[3] J. Lipman, H. Liu, I. Stojmenovic, Broadcasting in ad hoc networks, in: S. Misra, I. Woungang (Eds.), Guide to Wireless Ad Hoc Networks, Springer-Verlag, London, UK, 2009, pp.121-150 (Chapter 6).

[4] Tseng, Yu-Chee, et al. "The broadcast storm problem in a mobile ad hoc network", Wireless networks 8.2-3, pp.153-167, 2002.

[5] Wisitpongphan N, Tonguz OK, Parikh JS, Mudalige P, Bai F, Sadekar $\mathrm{V}$, "Broadcast storm mitigation techniques in vehicular ad hoc 
networks", Wireless Communications, IEEE vol. 14, no 6, pp. 84-94, 2007.

[6] Rakesh Kumar1 and Mayank Dave, "A Review of Various VANET Data Dissemination Protocols, " International Journal of $\mathrm{u}-$ and eService, and Technology Vol. 5, No. 3, September, 2012.

[7] Sooksan Panichpapiboon, Wasan Pattara-atikom, "A Review of Information Dissemination Protocols for Vehicular Ad Hoc Networks," IEEE Communications Surveys and Tutorials, vol. 14, no. 3, pp. 784798, Third Quarter, 2012.

[8] Chaqfeh, Moumena, Abderrahmane Lakas, and Imad Jawhar, "A survey on data dissemination in vehicular ad hoc networks," Vehicular Communications vol. 1, no 4, p. 214-225, 2014.

[9] Blum JJ, Eskandarian A (2009) Avoiding times slot boundary synchronization for multihop message broadcast in vehicular networks. In: IEEE 69th Vehicular Technology Conference (VTC Spring 2009), IEEE, pp 1-5.

[10] Schwartz RS, R Barbosa RR, Meratnia N, Heijenk G, Scholten H A directional data dissemination protocol for vehicular environments. Computer Communications 34(17):2057-207, 2011

[11] Schwartz, Ramon S., et al. "Exploiting beacons for scalable broadcast data dissemination in VANETs." Proceedings of the ninth ACM international workshop on Vehicular inter-networking, systems, and applications. ACM, 2012.
[12] Schwartz, Ramon S., Hans Scholten, and Paul Havinga. "A scalable data dissemination protocol for both highway and urban vehicular environments." EURASIP Journal on Wireless Communications and Networking 2013.1 (2013): 1-19.

[13] DW Kum, A Khan, YZ Cho, Traffic density-based broadcast scheme forvehicular ad hoc networks. IEICE Trans. Commun. E95B, 38753878 (2012)

[14] T Yu-Tian, J Rong-Hong, C Chien, W Chu-Fu, L Hsia-Hsin, A vehicledensity-based forwarding scheme for emergency message broadcasts in VANETs, in Proceedings of IEEE Mobile Adhoc and Sensor Systems (MASS) (San Francisco, CA, 2010), pp. 703-708

[15] Bhuiyan, Md Motaleb, Saad M. Salim, and Mohammad Rashedul Hasan. "Density aware broadcasting scheme for VANET." Communications (APCC), 2011 17th Asia-Pacific Conference on. IEEE, 2011.

[16] IEEE Standard for Information Technology-Telecommunications and Information Exchange Between Systems-Local and Metropolitan Area Networks-Specific Requirements Part 11, Wireless LAN Medium Access Control and Physical Layer Specifications, IEEE Standard 802.11p, 2010.

[17] NS3, "Network Simulator project",[Online]. Available: http://www.nsnam.org/

[18] SUMO, "Sumo - simulation of urban mobility." [Online]. Available: http://sumo.sourceforge.net/ 